\title{
Brd4 engagement from chromatin targeting to transcriptional regulation: selective contact with acetylated histone $\mathrm{H} 3$ and $\mathrm{H} 4$ Cheng-Ming Chiang
}

\author{
Address: Simmons Comprehensive Cancer Center, Department of Biochemistry, and Department of Pharmacology, University of Texas \\ Southwestern Medical Center, 5323 Harry Hines Boulevard, Dallas, Texas 75390, USA \\ Email: cheng-ming.chiang@utsouthwestern.edu
}

Fl000 Biology Reports 2009, I:98 (doi:I0.34I0/BI-98)

The electronic version of this article is the complete one and can be found at: http://F/000.com/Reports/Biology/content///98

\begin{abstract}
Bromodomain-containing protein 4 (Brd4) contains two tandem bromodomains (BDI and BD2) that bind preferentially to acetylated lysine residues found in histones and nonhistone proteins. This molecular recognition allows Brd4 to associate with acetylated chromatin throughout the cell cycle and regulates transcription at targeted loci. Recruitment of positive transcription elongation factor $b$, and possibly the general initiation cofactor Mediator as well, plays an important role in Brd4-regulated transcription. Selective contacts with acetyl-lysines in nucleosomal histones and chromatin-binding factors likely provide a molecular switch modulating the steps from chromatin targeting to transcriptional regulation, thus further expanding the 'acetylation code' for combinatorial regulation in eukaryotes.
\end{abstract}

\section{Introduction and context}

Bromodomain-containing protein 4 (Brd4), originally named MCAP (mitotic chromosome-associated protein) [1], belongs to a family of proteins with two aminoterminal tandem bromodomains (BD1 and BD2) and an extraterminal (ET) domain located either at its central region (for the longer isoform or family members) or close to the carboxyl terminus (for the shorter isoform or family members) (Figure 1). In addition to these three evolutionarily conserved domains, bromodomain-ET (BET) family proteins also contain other conserved regions [i.e., A, B, SEED (Ser/Glu/Asp-rich region), and carboxyl-terminal motif (CTM)] that are found in most but not all members and have mostly uncharacterized functions $[2,3]$. A unique feature of the BET proteins, which include yeast bromodomain factor 1 (Bdf1), bromodomain factor 2 (Bdf2), Drosophila female sterile homeotic (Fsh), and mammalian (e.g., human and mouse) Brd2, Brd3, Brd4, and testes/oocyte (i.e, germ cell)-specific Brdt (Figure 1), is their persistent association not only with interphase chromatin but also with mitotic chromosomes [4-7]. The association with mitotic chromosomes, also found for zebrafish and Xenopus Brd4 even before fish zygotic transcription is initiated [8], provides molecular marking for ensuing gene expression coinciding with or immediately following cell division [9-11].

Transcription-coupled mitotic control is likewise seen in viral systems. Several virus-encoded proteins, such as E2 transcription/replication factor encoded by BPV-1 (bovine papillomavirus type 1 ) and human papillomaviruses (HPVs), bind Brd4 directly to facilitate selective types of viral genome segregation during mitosis [12-15] and to generally regulate viral gene transcription during interphase [16-21]. Targeting cellular chromatin via direct association with a BET family protein seems to be widely used by other DNA tumor viruses for tethering viral genomes and exerting a dominant effect on viral and cellular gene expression [22-26]. Although viral genome partitioning also occurs through mitotic spindles/microtubules or specific regions of mitotic chromosomes in a Brd4-independent manner [27-29], attachment of viral genomes to cellular chromatin 
Figure I. Domain organization of the bromodomain and extraterminal domain (BET) family proteins

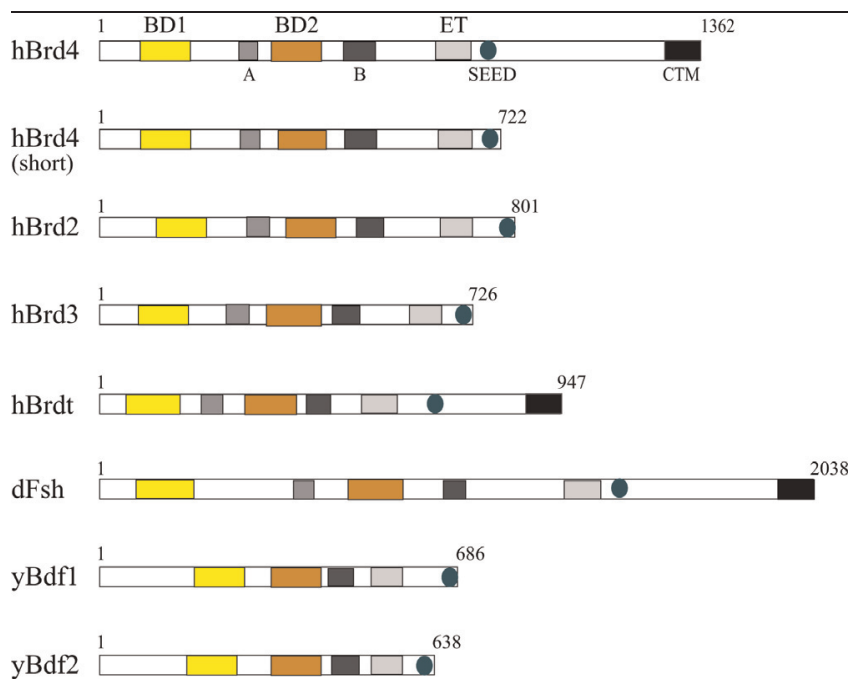

The evolutionarily conserved domains found in bromodomain-containing protein 4 (Brd4) and the other BET family proteins include bromodomain I (BDI), bromodomain 2 (BD2), extraterminal (ET), motif B, and SEED (Ser/ Glu/Asp-rich region). Motif $A$ and the carboxyl-terminal motif (CTM) are present only in some family members. Numbers indicate the amino acid boundaries of each protein derived from human (h), Drosophila (d), or yeast (y). The short form of human Brd4 (hBrd4) is also shown for comparison. Alignment of amino acid sequences and the accession number for each protein-coding gene are based on the information described by $\mathrm{Wu}$ and Chiang [3]. Bdf, bromodomain factor; Fsh, female sterile homeotic.

using BET chromatin adaptors provides a unique way for transcription-coupled genome dynamics to be regulated throughout the entire cell cycle.

Two ubiquitous transcription cofactors have been implicated in Brd4-mediated gene regulation (Figure 2). The general initiation cofactor Mediator (reviewed in [30]) was first identified by proteomic analysis of mouse Brd4 complexes [31] and later shown to associate with human Brd4 independently of the cyclin-dependent kinase 8 (Cdk8) module [3]. That Brd4 is not found in human transcription factor II D (TFIID) and is only detectable in some forms of human Mediator complexes [3] suggests that Brd4 has a unique role in the initiation of transcription that is yet to be elucidated. The other Brd4-associated general cofactor is positive transcription elongation factor b (P-TEFb) [31,32], a dimeric protein kinase consisting of Cdk9 and Cyclin T1 (or T2) that facilitates RNA polymerase II (Pol II)-dependent transcription through elongation blocks or pausing sites via phosphorylation at serine 2 (Ser2) of the carboxylterminal domain (CTD) in the largest subunit (RPB1) of Pol II (reviewed in [33-35]). Brd4 association with
$\mathrm{P}-\mathrm{TEFb}$ represents the active complex without the inhibitory components HEXIM $1 / 2$ and 7 SK small nuclear RNA. How Brd4 transits from chromatin targeting to transcriptional regulation in response to environmental cues is a crucial issue for our understanding of epigenetic control in eukaryotic transcription.

\section{Major recent advances}

Acetylation of histone H3 lysine 14 (H3K14ac) and H4 dual lysines - either K5 and K12, or K8 and K16 - seems to be crucial for Brd4 binding to acetylated histone peptides in vitro [5]. Whether the bromodomains of Brd4 also interact with acetyl-lysine in a nonhistone protein has recently been addressed by the identification of $\operatorname{Brd} 4$ as a bromodomain-containing protein that interacts with the RelA subunit of nuclear factor $(\mathrm{NF}) \kappa \mathrm{B}$ in an acetylation-dependent manner [36]. Both bromodomains of Brd4 are necessary for interacting with K310acetylated RelA and for tumor necrosis factor (TNF) $\alpha$ induced transcription of selective NFkB target genes, such as those encoding E-selectin and $\mathrm{TNF} \alpha$, but not A20. It is likely that association with acetylated RelA enhances the interaction between Brd4 and acetylated histone $\mathrm{H} 3$ and $\mathrm{H} 4$, which appears to be intrinsically weak and thus requires cooperative binding through multiple proteinprotein (Brd4-activator and Brd4-histone) and proteinDNA (activator-DNA) interactions [3]. This represents the commitment step for Brd4-regulated target gene transcription mediated by a transcriptional activator (Figure 2, left). Although subsequent recruitment of Mediator by Brd4 is likely necessary for Pol II entry to the promoter region and ensuing CTD phosphorylation (Figure 2, center), it is presently unknown how this step occurs in the context of chromatin. Very often, Pol II remains bound at the promoter region in an inactive state until stress-induced signals reactivate its elongation activity, allowing Pol II to pass through the pausing site.

A broader role of $\operatorname{Brd} 4$ in transcriptional regulation has likewise been addressed by knockdown studies and also with gene profiling and chromatin immunoprecipitation (ChIP) analysis of a large set of stress response genes and their chromatin marks. Zhou and colleagues [9] demonstrated that Brd4-mediated recruitment of P-TEFb to selective G1 genes encoding JunB, c-Myc, Cdk7, and Cyclin D1 begins in mid- and late anaphase, but prior to nuclear envelope formation and the entry of Pol II, TFIIB, and TFIIF, and continues into early G1 phase of the cell cycle. Interestingly, Brd4 association with mitotic chromosomes can occur at different stages of mitosis, depending on the cell type, and seems to be regulated by undefined cellular factors or covalent modifications. This mitotic association of selective G1 genes has been 
Figure 2. Acetylation of histone $\mathrm{H} 3$ and $\mathrm{H} 4$ lysine residues modulates Brd4 association with chromatin and the recruitment of Mediator and P-TEFb

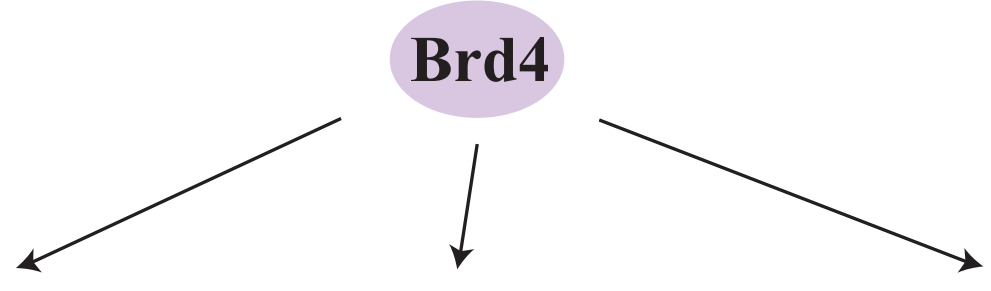

\section{Chromatin}

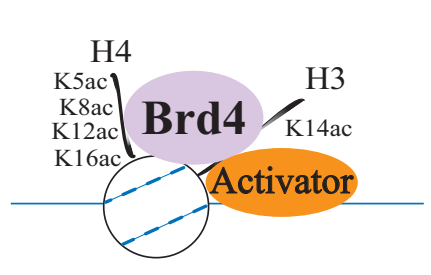

\section{Commitment}

Mediator

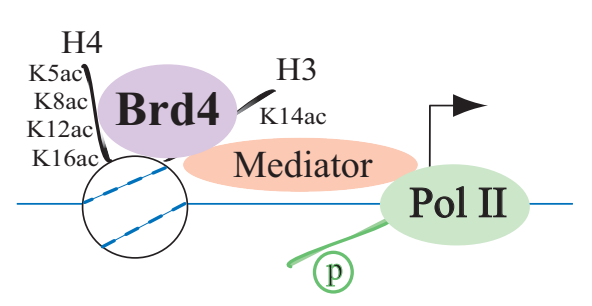

Initiation/post-initiation
P-TEFb

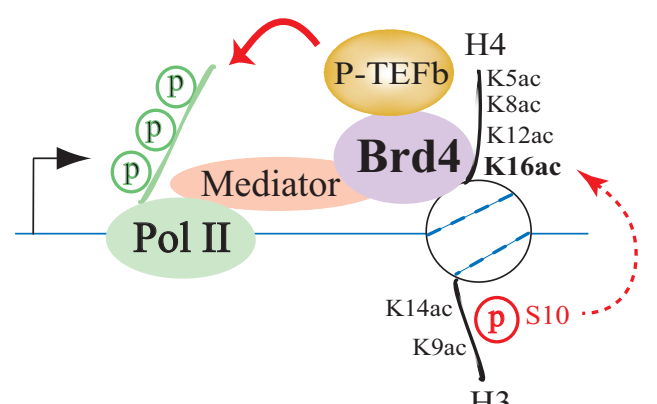

Elongation

Three steps for bromodomain-containing protein 4 (Brd4)-regulated chromatin targeting and transcriptional regulation are highlighted. The first step (left) represents a commitment to target gene transcription illustrated by cooperative binding between Brd4 and a transcriptional activator with acetylated chromatin through Brd4-activator interaction, activator-DNA contact, and Brd4 association, via its tandem bromodomains, with acetylated lysine 5 (K5ac), acetylated lysine 8 (K8ac), acetylated lysine I2 (KI2ac), and acetylated lysine I6 (KI6ac) of histone H4, and/or acetylated lysine I4 (KI4ac) of histone H3. The second step (center) is Brd4-mediated recruitment of the initiation cofactor Mediator to the promoter region, which often leads to phosphorylation of the RNA polymerase II (Pol II) carboxyl-terminal domain (CTD) at Ser5 during initiation and post-initiation events. The third step (right) is Brd4-facilitated recruitment of the elongation cofactor P-TEFb (positive transcription elongation factor b) to paused Pol II that results in Ser2 phosphorylation of the CTD, thereby allowing Pol II to resume elongation. The inducible recruitment of Brd4 to an acetylated nucleosome located downstream of the transcription start site (indicated by an arrow) appears to depend on crosstalk between acetylated lysine 9 (K9ac) and phosphorylated serine I0 (SI0) of $\mathrm{H} 3 \mathrm{mith} \mathrm{H} 4 \mathrm{KI}$ ac [38].

independently observed by the Ozato group, who elegantly illustrated by microarray analysis and quantitative RT-PCR (reverse transcription polymerase chain reaction) that many $\mathrm{G} 1$ gene products, including Cyclin $\mathrm{D} 1, \mathrm{D} 2, \mathrm{E} 1$, and E2, and phosphorylation of $\mathrm{pRb}$, are downregulated in Brd4-knockdown NIH3T3 cells and mouse embryonic fibroblasts [10], and that $\operatorname{Brd} 4$ marking of M/G1 genes increases significantly at telophase, coinciding with increased acetylation of histone $\mathrm{H} 3$ and $\mathrm{H} 4$ in these genes as well as P-TEFb recruitment and de novo M/G1 gene transcription [11].

The general significance of Brd4-recruited P-TEFb has been further revealed by another study using ChIP analysis of many primary response genes showing that, upon lipopolysaccharide stimulation of Toll-like receptor signaling, paused Pol II with Ser5 phosphorylation promptly resumes elongation following Ser2 phosphorylation of its CTD that is mediated by $\mathrm{P}-\mathrm{TEFb}$, which is recruited by Brd4 via recognition of induced acetylation at histone $\mathrm{H} 4 \mathrm{~K} 5 / 8 / 12$ in most primary response gene promoters [37]. Nevertheless, an independent study performed on the FOSL1 gene, which encodes the Fra1 subunit of AP-1 complexes, reported that MOF (males absent on the first)-acetylated H4K16 triggered by serum-induced PIM1 phosphorylation of H3 serine 10 (H3S10ph) are both required for Brd4 binding to targeted chromatin, which in turn recruits P-TEFb to the FOSL1 gene [38]. P-TEFb then induces Ser2 phosphorylation, which reactivates Pol II elongation activity (Figure 2, right). It appears that H3K9ac is needed for efficient H3S10ph to occur. While both reports clearly indicate the importance of $\mathrm{Brd} 4$ in recruiting $\mathrm{P}-\mathrm{TEFb}$ to poised Pol II in stress-induced gene activation, the nature of modified histone tails involved in the recruitment of $\operatorname{Brd} 4$ - that is, acetylated 
$\mathrm{H} 4 \mathrm{~K} 5 / 8 / 12$ versus $\mathrm{H} 3 \mathrm{~K} 9 \mathrm{acS} 10 \mathrm{ph}$-induced $\mathrm{H} 4 \mathrm{~K} 16 \mathrm{ac}$ - is different. This is possibly due to dynamic binding of different histone acetyltransferases (HATs) and histone deacetylases (HDACs) surrounding the targeted loci at the time of induction. A recent genome-wide binding survey of five HATs [p300, CREB-binding protein (CBP), p300/CBP-associated factor (PCAF), MOF, and Tip60] and four HDACs (HDAC1, HDAC2, HDAC3, and HDAC6) by high-throughput sequencing of antibodypulled down ChIP fragments (ChIP-Seq) isolated from resting and induced primary human $\mathrm{CD} 4^{+} \mathrm{T}$ cells surprisingly revealed that both HATs and HDACs coreside in most active genes and also in many repressed but inducible genes [39]. Since H3K9/14 and H4K5/8/ 12/16 can be acetylated by various HATs (p300/CBP for H3K14 and H4K5/8, PCAF for H3K9/14, Tip60 for H3K14 and H4K5/8/12/16, and MOF for H4K16) (reviewed in $[40,41]$ ), the distinct patterns of acetylated residues identified in Brd4 recruitment probably reflect specific HATs and HDACs available in the surveyed cell types and the induced signaling pathways. This intriguing possibility needs to be explored in the future.

Brd4-mediated recruitment of P-TEFb is also important for transcription of viral promoters, as recently demonstrated in several HPV-harboring human cell lines, in which HPV-encoded E2 protein is able to suppress viral transcription by displacing P-TEFb from Brd4-bound HPV chromatin owing to competition between highaffinity E2 and low-affinity P-TEFb for the same binding site at the CTM of Brd4 [42]. This result provides an additional mechanism for the E2-Brd4 silencing complex that efficiently inhibits HPV chromatin transcription by blocking TFIID and Pol II recruitment to the viral promoter in a bromodomain-dependent manner [16]. In HPV it is likely that both Brd4-regulated initiation and elongation steps of transcription are simultaneously targeted by E2 for efficient suppression of viral gene expression, consistent with a central role of Brd4 in both cellular and viral gene transcription.

Recent structural determination of several BET bromodomains further provides atomic details for bromodomain/acetyl-lysine interactions. While an earlier study reporting that homodimer formation of $\mathrm{BD} 1$ derived from human Brd 2 creates a negatively charged secondary binding pocket at the dimer interface may help to determine binding specificity provided by the acetyllysine-binding pocket of BD1 [43], dimerization of mouse Brd4 BD1 or BD2 was not seen in solution or in crystal structures [44]. Both BD1 and BD2 of mouse Brd4 exhibit the classical bromodomain fold consisting of four $\alpha$-helices $\left(\alpha_{Z}, \alpha_{A}, \alpha_{B}\right.$, and $\left.\alpha_{C}\right)$ and two interconnecting ZA and BC loops. Charged amino acid residues (acidic in $\mathrm{BD} 2$, basic in $\mathrm{BD} 1$ ) surrounding the acetyl-lysine-binding pocket may enhance the binding specificity of BD1 and BD2. An amino-terminal extension of $\mathrm{BD} 1$ that helps stabilize the bromodomain fold is also evident in the structure. It is noted that the binding affinities of BD2 for K390-acetylated Cyclin T1 peptide and $\mathrm{H} 4 \mathrm{~K} 5 \mathrm{ac}$ or $\mathrm{H} 4 \mathrm{~K} 16 \mathrm{ac}$ peptide are rather similar (Kd $\sim 110 \mu \mathrm{M}$ ), indicating the possibility of switching of BD2 binding from acetylated chromatin to acetylated $\mathrm{P}-\mathrm{TEFb}$ [44]. As predicted [3], cooperative binding between Brd4 and a DNA-binding factor is generally required to stabilize Brd4 association with chromatin, as now shown by the low affinity measured for the interaction between BD1/BD2 and acetylated histone tails, which is mediated by a few hydrogen bonds and sparse hydrophobic contacts [44].

The cooperative nature of bromodomain binding to acetyl-lysine has also been demonstrated by isothermal calorimetry measuring differences in the binding affinities of BD1 and BD2 of mouse Brdt for histone H3 and H4 peptides with varying degrees of acetylation at distinct lysine residues [45]. Surprisingly, BD2 fails to bind tetra- and mono-acetylated $\mathrm{H} 4$ peptides, whereas BD1 binds as efficiently as an amino-terminal portion of Brdt protein containing both BD1 and BD2 to tetraacetylated $\mathrm{H} 4$ peptide ( $\mathrm{Kd} \sim 28.0 \mu \mathrm{M})$. Clearly, BD1 and $\mathrm{BD} 2$ are not equivalent binders and each has unique features and binding affinities for acetyl-lysine residues in both histones and nonhistone proteins. A compilation of binding studies performed with various BET proteins or their derived bromodomains and acetylated lysines in histone $\mathrm{H} 3$ and $\mathrm{H} 4$ at different positions is summarized in Figure 3. Clearly, cooperative binding to acetylated histone tails requires diacetylated lysines with properly spaced residues. A crystal structure of mouse Brdt BD1 in complex with diacetylated $\mathrm{H} 4 \mathrm{~K} 5 \mathrm{acK} 8 \mathrm{ac}$ peptide revealed an unexpected association of two acetylation marks on the same histone tail with a single bromodomain [45]. $\mathrm{BD} 1$ binding to $\mathrm{K} 5 \mathrm{ac}$ is prototypical and K8ac-BD1 interaction is mediated primarily through hydrophobic contacts, mimicking overall a two-pronged plug with K5ac inserted deep into the acetyl-lysine-binding pocket and K8ac bent diagonally towards K5ac. The structure of $\mathrm{BD} 1$ binding to the diacetylated K5ac-GG-K8ac peptide seems to be conserved among several other bromodomains, although these are yet to be experimentally illustrated.

\section{Future directions}

Recent structural determination and functional assays from both gene/domain-based and global approaches have shed light on the cooperative nature of interactions between the bromodomains of BET family proteins and 
Figure 3. Summary of binding studies performed with various BET family proteins or their derived bromodomains with acetylated histone $\mathrm{H} 3$ and $\mathrm{H} 4$ peptides in vitro or with chromatin in live cells

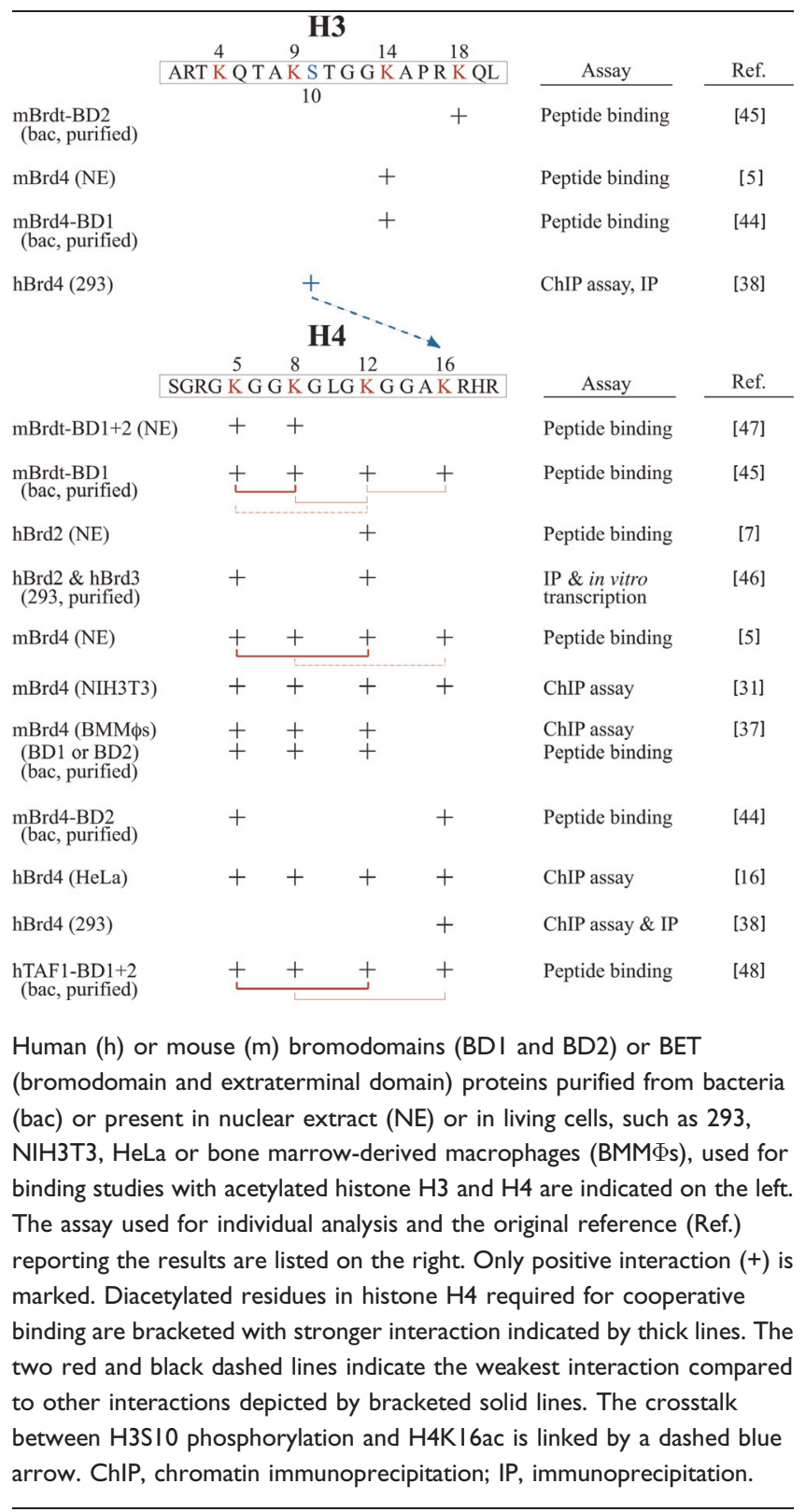

acetylated histone and nonhistone proteins. The diverse recognition of acetylated lysine residues in histone H3 and $\mathrm{H} 4$ at distinct positions likely reflects the transition from chromatin targeting to transcriptional regulation and the need to break and reform the contact between two tandem bromodomains and acetyl-lysine residues. Elucidation of the combinatorial usage of acetylation codes at different stages of chromatin binding and transcriptional events will need to be conducted in a variety of cell types under both unstressed and stressed conditions in the future. The complexity of cellular environments also points to the need for applying welldefined reconstitution systems to elucidate the molecular and biochemical pathways leading to differential utilization of different BET family proteins in chromatin targeting and gene regulation. While the functional analysis of Brd4-regulated P-TEFb recruitment in diverse gene responses represents a continuing interest for future studies, the role of Mediator and its mechanistic involvement in BET-mediated transcription needs to be established. The biological effects of protein domains in BET family members other than the two bromodomains also require detailed characterization. Without doubt, the identification of chromatin adaptors implicated in transcriptional regulation and the elucidation of the acetylation code warrant continuing efforts for future investigations.

\section{Abbreviations}

ac, acetylation; BD, bromodomain; BET, bromodomain and extraterminal domain; Brd4, bromodomain-containing protein 4; CBP, CREB-binding protein; $\mathrm{Cdk}$, cyclin-dependent kinase; ChIP, chromatin immunoprecipitation; CTD, carboxyl-terminal domain; CTM, carboxyl-terminal motif; ET, extraterminal; HAT, histone acetyltransferase; HDAC, histone deacetylase; HPV, human papillomavirus; MOF, males absent on the first; NF, nuclear factor; PCAF, p300/CBP-associated factor; ph, phosphorylation; Pol II, RNA polymerase II; P-TEFb, positive transcription elongation factor $\mathrm{b}$; SEED, Ser/ Glu/Asp-rich region; TFIID, transcription factor II D; TNF, tumor necrosis factor.

\section{Competing interests}

The author declares that he has no competing interests.

\section{Acknowledgments}

I thank Dr Shwu-Yuan Wu for help with the illustrations and for providing comments on the manuscript. The research in my laboratory is currently supported by grants CA103867 and CA124760 from the National Institutes of Health (Bethesda, MD, USA). This work is Report CSCN \#055 from the Harold C Simmons Comprehensive Cancer Center at the University of Texas Southwestern Medical Center.

\section{References}

I. Dey A, Ellenberg J, Farina A, Coleman AE, Maruyama T, Sciortino S, Lippincott-Schwartz J, Ozato K: A bromodomain protein, MCAP, associates with mitotic chromosomes and affects $G(2)$-to-M transition. Mol Cell Biol 2000, 20:6537-49.

2. Florence B, Faller DV: You bet-cha: a novel family of transcriptional regulators. Front Biosci 200I, 6:D1008-18. 
3. Wu S-Y, Chiang C-M: The double bromodomain-containing chromatin adaptor Brd4 and transcriptional regulation. J Biol Chem 2007, 282:|3|4|-5.

4. Chua P, Roeder GS: BdfI, a yeast chromosomal protein required for sporulation. Mol Cell Biol 1995, 15:3685-96.

5. Dey A, Chitsaz F, Abbasi A, Misteli T, Ozato K: The double bromodomain protein Brd4 binds to acetylated chromatin during interphase and mitosis. Proc Natl Acad Sci U S A 2003, 100:8758-63.

6. Pivot-Pajot C, Caron C, Govin J, Vion A, Rousseaux S, Khochbin S: Acetylation-dependent chromatin reorganization by BRDT, a testis-specific bromodomain-containing protein. Mol Cell Biol 2003, 23:5354-65.

7. Kanno T, Kanno Y, Siegel RM, Jang MK, Lenardo MJ, Ozato K: Selective recognition of acetylated histones by bromodomain proteins visualized in living cells. Mol Cell 2004, I3:33-43.

FI000 Factor 6.0 Must Read

Evaluated by Cheng-Ming Chiang 05 Feb 2004

8. Toyama R, Rebbert ML, Dey A, Ozato K, Dawid IB: Brd4 associates with mitotic chromosomes throughout early zebrafish embryogenesis. Dev Dyn 2008, 237:1636-44.

9. Yang Z, He N, Zhou Q: Brd4 recruits P-TEFb to chromosomes at late mitosis to promote $\mathbf{G I}$ gene expression and cell cycle progression. Mol Cell Biol 2008, 28:967-76.

FI000 Factor 3.0 Recommended

Evaluated by Cheng-Ming Chiang 16 Apr 2008

10. Mochizuki K, Nishiyama A, Jang MK, Dey A, Ghosh A, Tamura T, Natsume H, Yao H, Ozato K: The bromodomain protein Brd4 stimulates $\mathrm{GI}$ gene transcription and promotes progression to S phase. J Biol Chem 2008, 283:9040-8.

FI000 Factor 3.0 Recommended

Evaluated by Cheng-Ming Chiang 17 Apr 2008

II. Dey A, Nishiyama A, Karpova T, McNally J, Ozato K: Brd4 marks select genes on mitotic chromatin and directs postmitotic transcription. Mol Biol Cell 2009, 20:4899-909.

12. You J, Croyle JL, Nishimura A, Ozato K, Howley PM: Interaction of the bovine papillomavirus E2 protein with Brd4 tethers the viral DNA to host mitotic chromosomes. Cell 2004, I I 7:349-60.

FI000 Factor 6.0 Must Read

Evaluated by Cheng-Ming Chiang 07 May 2004

13. McPhillips MG, Ozato K, McBride AA: Interaction of bovine papillomavirus E2 protein with Brd4 stabilizes its association with chromatin. J Virol 2005, 79:8920-32.

14. Parish JL, Bean AM, Park RB, Androphy EJ: ChIRI is required for loading papillomavirus E2 onto mitotic chromosomes and viral genome maintenance. Mol Cell 2006, 24:867-76.

FI000 Factor 6.0 Must Read

Evaluated by Cheng-Ming Chiang 10 Jan 2007

15. Abbate EA, Voitenleitner C, Botchan MR: Structure of the papillomavirus DNA-tethering complex E2:Brd4 and a peptide that ablates HPV chromosomal association. Mol Cell 2006, 24:877-89.

FI000 Factor 6.0 Must Read

Evaluated by Cheng-Ming Chiang 10 Jan 2007

16. Wu S-Y, Lee AY, Hou SY, Kemper JK, Erdjument-Bromage H, Tempst P, Chiang C-M: Brd4 links chromatin targeting to HPV transcriptional silencing. Genes Dev 2006, 20:2383-96.

17. Ilves I, Mäemets K, Silla T, Janikson K, Ustav M: Brd4 is involved in multiple processes of the bovine papillomavirus type I life cycle. J Virol 2006, 80:3660-5.

18. Schweiger MR, You J, Howley PM: Bromodomain protein 4 mediates the papillomavirus E2 transcriptional activation function. J Virol 2006, 80:4276-85.
19. McPhillips MG, Oliveira JG, Spindler JE, Mitra R, McBride AA: Brd4 is required for E2-mediated transcriptional activation but not genome partitioning of all papillomaviruses. J Virol 2006, 80:9530-43.

20. Sénéchal H, Poirier GG, Coulombe B, Laimins LA, Archambault J: Amino acid substitutions that specifically impair the transcriptional activity of papillomavirus E2 affect binding to the long isoform of Brd4. Virology 2007, 358:10-7.

21. Lee A-Y, Chiang C-M: Chromatin adaptor Brd4 modulates E2 transcription activity and protein stability. J Biol Chem 2009, 284:2778-86.

22. You J, Srinivasan V, Denis GV, Harrington WJ Jr, Ballestas ME, Kaye KM, Howley PM: Kaposi's sarcoma-associated herpesvirus latency-associated nuclear antigen interacts with bromodomain protein Brd4 on host mitotic chromosomes. J Virol 2006, 80:8909-19.

23. Viejo-Borbolla $A$, Ottinger $M$, Brüning $E$, Bürger $A$, König R, Kati $E$, Sheldon JA, Schulz TF: Brd2/RING3 interacts with a chromatinbinding domain in the Kaposi's Sarcoma-associated herpesvirus latency-associated nuclear antigen I (LANA-I) that is required for multiple functions of LANA-I. J Virol 2005, 79:13618-29.

24. Ottinger M, Christalla T, Nathan K, Brinkmann MM, Viejo-Borbolla A Schulz TF: Kaposi's sarcoma-associated herpesvirus LANA-I interacts with the short variant of BRD4 and releases cells from a BRD4- and BRD2/RING3-induced GI cell cycle arrest. J Virol 2006, 80: 10772-86.

25. Lin A, Wang S, Nguyen T, Shire K, Frappier L: The EBNAI protein of Epstein-Barr virus functionally interacts with Brd4. J Virol 2008, 82:12009-19.

26. Ottinger M, Pliquet D, Christalla T, Frank R, Stewart JP, Schulz TF: The interaction of the gammaherpesvirus 68 orf 73 protein with cellular BET proteins affects the activation of cell cycle promoters. J Virol 2009, 83:4423-34.

27. Van Tine BA, Dao LD, Wu S-Y, Sonbuchner TM, Lin BY, Zou N Chiang C-M, Broker TR, Chow LT: Human papillomavirus (HPV) origin-binding protein associates with mitotic spindles to enable viral DNA partitioning. Proc Natl Acad Sci U S A 2004, 101:4030-5.

28. Dao LD, Duffy A, Van Tine BA, Wu S-Y, Chiang C-M, Broker TR, Chow LT: Dynamic localization of the human papillomavirus type II origin binding protein E2 through mitosis while in association with the spindle apparatus. J Virol 2006, 80:4792-800.

29. Poddar A, Reed SC, McPhillips MG, Spindler JE, McBride AA: The human papillomavirus type 8 E2 tethering protein targets the ribosomal DNA loci of host mitotic chromosomes. J Virol 2009, 83:640-50.

30. Thomas MC, Chiang C-M: The general transcription machinery and general cofactors. Crit Rev Biochem Mol Biol 2006, 4I: 105-78.

31. Jang MK, Mochizuki K, Zhou M, Jeong HS, Brady JN, Ozato K: The bromodomain protein $\mathrm{Brd4}$ is a positive regulatory component of $\mathrm{P}$-TEFb and stimulates RNA polymerase II-dependent transcription. Mol Cell 2005, 19:523-34.

FI000 Factor 6.0 Must Read

Evaluated by Cheng-Ming Chiang 02 Sep 2005

32. Yang Z, Yik JH, Chen R, He N, Jang MK, Ozato K, Zhou Q: Recruitment of P-TEFb for stimulation of transcriptional elongation by the bromodomain protein Brd4. Mol Cell 2005, 19:535-45.

FI000 Factor 6.0 Must Read

Evaluated by Cheng-Ming Chiang 02 Sep 2005

33. Peterlin $\mathrm{BM}$, Price $\mathrm{DH}$ : Controlling the elongation phase of transcription with P-TEFb. Mol Cell 2006, 23:297-305.

34. Zhou Q, Yik JH: The Yin and Yang of P-TEFb regulation: implications for human immunodeficiency virus gene expression and global control of cell growth and differentiation. Microbiol Mol Biol Rev 2006, 70:646-59. 
35. Brès $\mathrm{V}$, Yoh SM, Jones KA: The multi-tasking P-TEFb complex. Curr Opin Cell Biol 2008, 20:334-40.

36. Huang B, Yang X-D, Zhou M-M, Ozato K, Chen L-F: Brd4 coactivates transcriptional activation of NF-kappaB via specific binding to acetylated RelA. Mol Cell Biol 2009, 29:1375-87.

FI000 Factor 3.0 Recommended

Evaluated by Cheng-Ming Chiang 18 Feb 2009

37. Hargreaves DC, Horng T, Medzhitov R: Control of inducible gene expression by signal-dependent transcriptional elongation. Cell 2009, 138:129-45.

FI000 Factor 3.0 Recommended

Evaluated by Andrew D Sharrocks 26 Nov 2009

38. Zippo A, Serafini R, Rocchigiani M, Pennacchini S, Krepelova A, Oliviero S: Histone crosstalk between H3SIOph and H4KI Gac generates a histone code that mediates transcription elongation. Cell 2009, I38: I |22-36.

39. Wang Z, Zang C, Cui K, Schones DE, Barski A, Peng W, Zhao K: Genome-wide mapping of HATs and HDACs reveals distinct functions in active and inactive genes. Cell 2009, 138:1019-31.

FI000 Factor 4.8 Must Read

Evaluated by Jeffrey Hayes 25 Sep 2009, Patrick Matthias 08 Oct 2009

40. Kouzarides T: Chromatin modifications and their function. Cell 2007, I 28:693-705.

41. Rea S, Xouri G, Akhtar A: Males absent on the first (MOF): from flies to humans. Oncogene 2007, 26:5385-94.
42. Yan J, Li Q, Lievens S, Tavernier J, You J: Abrogation of the Brd4P-TEFb positive transcription complex by the papillomavirus E2 protein contributes to the viral oncogene repression. J Virol 2009, [Epub ahead of print].

43. Nakamura $Y$, Umehara T, Nakano K, Jang MK, Shirouzu M, Morita S, Uda-Tochio H, Hamana H, Terada T, Adachi N, Matsumoto T, Tanaka A, Horikoshi M, Ozato K, Padmanabhan B, Yokoyama S: Crystal structure of the human BRD2 bromodomain: insights into dimerization and recognition of acetylated histone $\mathrm{H} 4$. J Biol Chem 2007, 282:4193-201.

44. Vollmuth F, Blankenfeldt W, Geyer M: Structures of the dual bromodomains of the P-TEFb activating protein Brd4 at atomic resolution. J Biol Chem 2009, [Epub ahead of print].

45. Morinière J, Rousseaux $S$, Steuerwald $U$, Soler-López $M$, Curtet $S$, Vitte AL, Govin J, Gaucher J, Sadoul K, Hart DJ, Krijgsveld J, Khochbin S, Müller CW, Petosa C: Cooperative binding of two acetylation marks on a histone tail by a single bromodomain. Nature 2009, 461:664-8.

46. LeRoy G, Rickards B, Flint SJ: The double bromodomain proteins Brd2 and Brd3 couple histone acetylation to transcription. Mol Cell 2008, 30:5I-60.

FI000 Factor 6.0 Must Read Evaluated by Cheng-Ming Chiang 16 Apr 2008

47. Sasaki K, Ito T, Nishino N, Khochbin S, Yoshida M: Real-time imaging of histone $\mathbf{H} 4$ hyperacetylation in living cells. Proc Natl Acad Sci U S A 2009, 106:16257-62.

48. Jacobson RH, Ladurner AG, King DS, Tjian R: Structure and function of a human $\mathrm{TAF}_{||} \mathbf{2 5 0}$ double bromodomain module. Science 2000, 288: 1422-5. 